\title{
LHC PILOT BUNCHES FROM THE CERN PS BOOSTER
}

\author{
M. Benedikt, CERN, Geneva, Switzerland
}

\begin{abstract}
For the first commissioning phase of the LHC, a single proton bunch is required. The production of this so-called "LHC pilot bunch" will follow a different scheme than the one of the nominal LHC proton bunch train. Both the transverse and the longitudinal LHC bunch characteristics should already be established in the PS Booster. The parameter space for the LHC pilot bunch spans a factor 66 in beam brightness. The intensity should be variable by a factor of 22 from $5.0 \cdot 10^{9}$ to $1.1 \cdot 10^{11}$, covering the range from the LHC quench limit up to the intensity of a nominal LHC bunch. The transverse emittances (at Booster ejection) should be adjustable in the range $0.85 \pi \mu \mathrm{m} \leq \varepsilon_{\text {norm,rms }} \leq 2.5 \pi \mu \mathrm{m}$, while the longitudinal emittance is kept constant at $0.3 \mathrm{eVs}$, slightly below the nominal one $(0.35 \mathrm{eVs})$. To cover the whole parameter space, a mixture of several ingredients was required: intensity adjustment with low voltage rf-capture; definition of the transverse emittance with shavers; controlled blow-up followed by longitudinal shaving to define the longitudinal emittance. All beam variants were produced on harmonic two, with only one bunch being sent to the downstream accelerator chain. To cover also the lowest intensity side, the Linac beam was reduced by a factor 5 with a "sieve". The pilot bunches corresponding to the "corners" of the parameter space and a few selected inner reference points were successfully produced in the PS Booster.
\end{abstract}

\section{INTRODUCTION}

The PS-Booster (PSB) is the first circular machine in the LHC proton injection chain. For production of the nominal LHC beam [1], a horizontal three-turn injection is used to accumulate the $50 \mathrm{MeV}$ Linac beam. The multiturn injection process in the PSB determines the transverse emittances of the beam and therefore the final LHC beam brightness. On the contrary, the longitudinal characteristic of the nominal LHC beam is only fixed at ejection from the PS. Complicated RF gymnastics (triple splitting from harmonics 7 to 21 at injection and two double splittings from harmonics 21 to 42 and 84 before ejection), employing also some controlled longitudinal beam blow-up, are performed in the PS to generate the nominal bunch train for the LHC [2].

The situation is rather different for the first commissioning phase of the LHC, where only a single proton bunch will be required. The production of this pilot bunch will follow a completely different scheme than the production of the nominal LHC proton bunch train. Not only the transverse but also the longitudinal bunch characteristics will have to be established in the

Michael.Benedikt@cern.ch
PSB thus avoiding all longitudinal gymnastics in the PS. After ejection from the PSB, the pilot bunch should be passed on to the LHC by the downstream machines with the main concerns being to keep the bunch characteristics unchanged and to minimize longitudinal and transverse blow-ups.

\section{PARAMETER SPACE}

At present the various strategies for LHC start-up and the first commissioning phase with beam are still being discussed and consequently no definite specifications for an LHC pilot bunch have been formulated. One of the main goals was therefore to develop a flexible strategy for pilot bunch production that allows a large variation of beam parameters to be best adapted to whatever will be requested by the LHC. For an estimate of the required parameter space some LHC key figures were used:

- Intensity: The number of protons in the bunch should be variable from $5.0 \cdot 10^{9}$ to $1.1 \cdot 10^{11}$. The lower figure corresponds to the LHC quench limit at injection energy $(450 \mathrm{GeV})$ and the higher is the intensity of a nominal bunch.

- Transverse emittance: Normalised horizontal and vertical rms emittances should be adjustable in the range from $0.85 \pi \mu \mathrm{m}$ to $2.5 \pi \mu \mathrm{m}$ at PSB ejection energy of $1.4 \mathrm{GeV}$. The $2.5 \pi \mu \mathrm{m}$ correspond to the nominal emittance of the LHC beam (3.5 $\pi \mu \mathrm{m}$ at LHC injection). The emittance variation should allow aperture scans, orbit adjustments and first dynamic aperture studies.

- Longitudinal emittance: This is the only pilot bunch parameter that is foreseen to be kept constant at $0.3 \mathrm{eVs}$ (at PSB ejection), which is just slightly below the nominal longitudinal emittance of $0.35 \mathrm{eVs}$ (in the LHC) thus allowing for some additional blow up in the injector chain.

Combining the above requirements of an intensity variation by a factor 22 and a transverse emittance variation by a factor 3 shows that the parameter space for the LHC pilot bunch spans a factor 66 in beam brightness defined as ratio of protons per bunch and normalized emittance, $N_{\mathrm{b}} / \varepsilon_{\mathrm{n}}$. A graphical representation of the parameter space is given in Figure 1.

The production of the pilot bunches is further complicated by the fact that neither the required low bunch intensities nor the longitudinal emittance of $0.3 \mathrm{eVs}$ correspond to the typical working range of the PSB. In standard operation beams with up to $1 \cdot 10^{13}$ protons per bunch and longitudinal emittances of typically $1.5 \mathrm{eVs}$ are produced. 


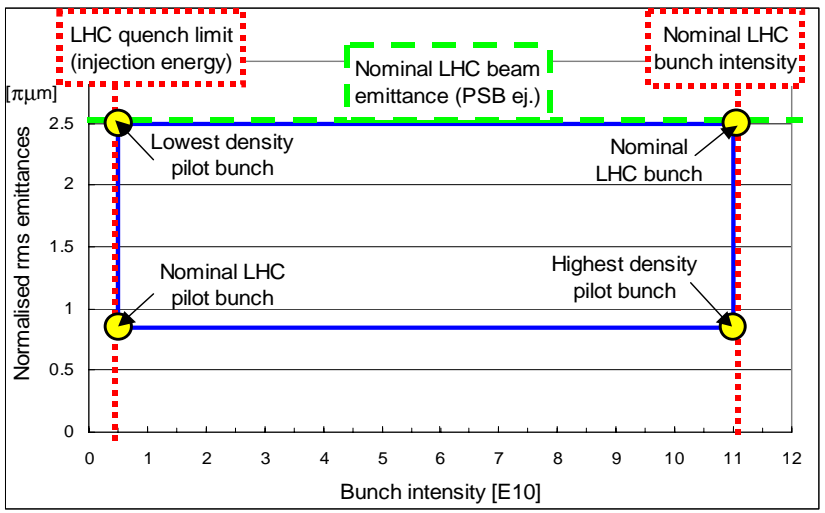

Figure 1: LHC pilot bunch parameter space.

\section{STRATEGY FOR PILOT PRODUCTION}

During 2002 several machine development sessions on the subject of LHC pilot bunch production were performed on the PSB. The first step, before any detailed optimization work could start, was to develop a strategy enabling to produce and to change the very different beam variants within a reasonable amount of time. To cover the whole pilot beam parameter space, a mixture of several ingredients was required.

\section{Longitudinal emittance}

The control of this parameter is straightforward and the required value of $0.3 \mathrm{eVs}$ was achieved by longitudinal shaving. For this the rf voltage was reduced to limit the bucket acceptance of $0.3 \mathrm{eVs}$ approximately in the middle of acceleration. Afterwards the rf voltage is again raised to the nominal $8 \mathrm{kV}$ required for a correct longitudinal matching with the PS rf system.

\section{Transverse emittances}

Independent horizontal and vertical "shavers" are used for emittance control in the PSB. For each plane the system uses a single correction dipole to deflect the orbit towards an aperture limitation where the beam is shaved in betatron amplitudes. This process is applied directly after $\mathrm{rf}$ capture at the beginning of acceleration. At this moment, there is almost no adiabatic damping and the physical beam size is still large, improving the precision of the shaving.

\section{Intensity (beam brightness)}

Variation of the beam brightness by a factor 66 is the most challenging issue; for every transverse emittance value established by beam shaving, an additional factor 22 in intensity variation is required. For this a combination of three different techniques was used.

1. The longitudinal phase space density was controlled in two steps. Firstly the rf voltage during the bunching process was varied hereby changing the part of the unbunched linac beam that is captured and accelerated. Secondly controlled longitudinal blow up was applied during the first half of the acceleration cycle to depopulate the longitudinal phase space. This was done with a higher harmonic rf system [3]. The final longitudinal emittance is then fixed afterwards by shaving with the principal rf system. Applying more or less blow up gives a good control of the longitudinal density and, since there is quasi no coupling between transverse and longitudinal phase spaces in the $\mathrm{PSB}$, the effect is equivalent to a change of the transverse beam brightness. In this way an intensity (brightness) variation of up to a factor 4 could be achieved.

2. The (transverse) brightness of the beam in the PSB can be influenced by the settings used for the multi turn injection process. The most effective parameters are the steering and the betatron matching of the injection line, the horizontal and vertical tunes of the machine and the fine timing of the "slow" injection bumpers. A brightness variation of a factor 2 was achieved with the different settings.

3. The intensity (brightness) of the linac beam can be changed by a sharp factor 5 . This was done with the so-called "sieve" which is a mechanical device installed in the injection line that allows only about $20 \%$ of the beam to pass through.

Combining the first two techniques allows continuous variation of the beam intensity by a factor 8 , applying the multiplicative factor 5 provided by the "sieve" intensity reduction allows to cover the complete LHC pilot bunch parameter space. The upper part of Figure 2 shows the voltages of second harmonic and blow-up rf systems for generation of the $5.0 \cdot 10^{9}-0.85 \pi \mu \mathrm{m}$ pilot bunch along the acceleration cycle. The effects of capture voltage, beam blow up, transverse and longitudinal shavings on the evolution of the beam current are shown in the lower part.

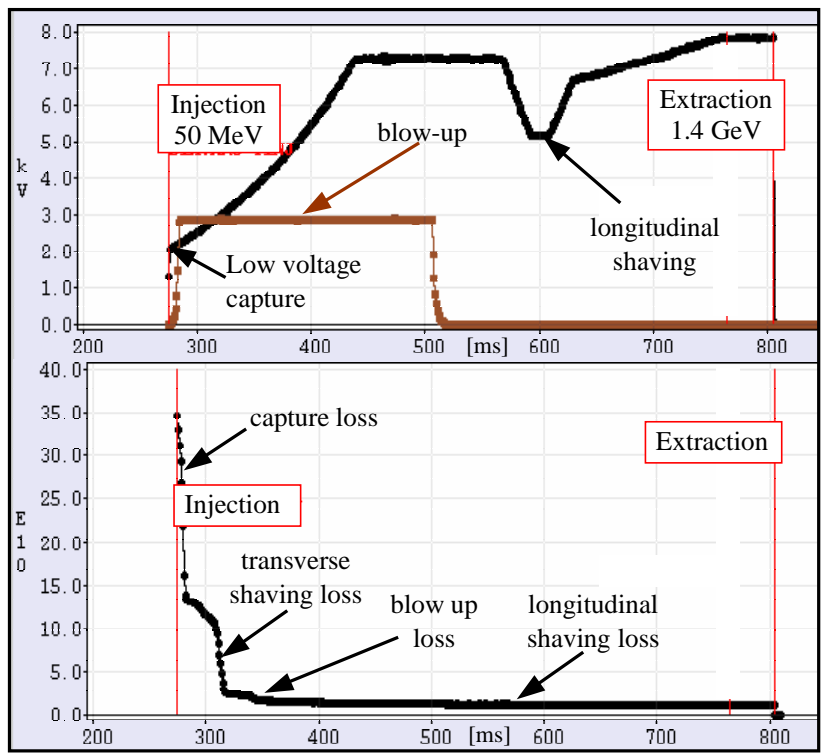

Figure 2: RF voltages and beam intensity along the cycle. 


\section{Beam loss issues}

The maximum loss when producing pilot bunches with the described strategy amounts to about $10^{12}$ protons per cycle where most of the loss occurs at injection energy. This is to be compared with beam losses in the order of $10^{13}$ protons per cycle for day-to-day high intensity operation.

\section{BEAM TESTS}

\section{Machine set up}

All beam tests were performed in ring 3 of the PSB. A three-turn injection, similar to the one used for the nominal LHC beam, was used as starting point for the setup. Experience during the last years has shown that this type of injection is best suited for a good reproducibility and stability which is essential for both the nominal LHC and the pilot bunch beams. Resonance compensation and working point optimization are less of an issue because the (vertical) incoherent space charge tune spread at injection is significantly smaller than in the case of the nominal beam where $\Delta Q_{\mathrm{v}} \leq-0.45$. In contrast to the nominal beam all pilot bunch variants were produced with the second harmonics rf system. Obviously this generates two pilot bunches but only one is being sent to the downstream accelerator chain. The use of $h=2$ for acceleration has two advantages from the operational point of view. The second harmonics $\mathrm{rf}$ system is naturally better suited to the production of a relatively small longitudinal emittance (first and second harmonics systems have the same nominal voltage of $8 \mathrm{kV}$ ) and by accelerating two bunches, the intensity in the machine is doubled hereby relaxing the demands on the dynamic range of various control loops.

\section{Results}

To validate the proposed strategy, pilot bunches corresponding to the corners of the parameter space and a few selected reference points were produced in ring 3 of the PSB. Pilot bunches exist in two flavors: one group, with intensities between $5 \cdot 10^{9}$ and $2.0 \cdot 10^{10}$ was fabricated by using the sieve for Linac beam intensity reduction, the other group with intensities between $2.5 \cdot 10^{10}$ and $1.1 \cdot 10^{11}$ was based on the nominal Linac beam. Figure 3 shows the LHC pilot bunch parameter space, the reference beams that were produced in 2002 are indicated as large spots.

It is interesting to note that the bunch corresponding to the bottom right corner in Figure 3 features an incoherent space charge tune shift of $\Delta Q_{\mathrm{v}} \approx-0.13$ at SPS injection. This is three times the tune shift of a nominal LHC bunch and might be at the limit of what can be accepted by the SPS.

The intensities were observed to be stable on all bunch variants to better than $\pm 10 \%$, corresponding to LHC demands. It is worth looking at the figures in a bit more detail: $\pm 10 \%$ of the lowest intensity pilot bunch corresponds to a fluctuation below $\pm 5 \cdot 10^{8}$ protons. This is a factor $5 \cdot 10^{-5}$ smaller than the maximum intensity of
$10^{13}$ protons that can be obtained from ring 3 , underlining the versatility of the PSB.

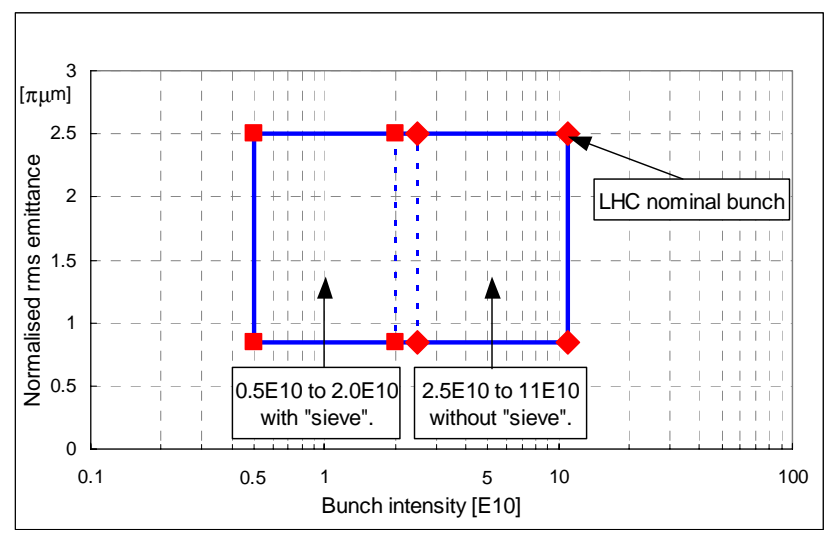

Figure 3: LHC pilot bunches produced in the PSB.

\section{CONCLUSIONS}

For the first commissioning phase of the LHC a single bunch will be required. All beam parameters of this pilot bunch should already be defined and fixed at ejection from the PSB in contrast to the production scheme for the nominal LHC beam, where all the longitudinal characteristics is established in the PS. Presently the requirements for the LHC pilot bunch are not fixed; for this reason a relatively large parameter space was defined using critical LHC parameters as input. This parameter space spans a factor 66 in beam brightness and an operational strategy for the PSB was developed in order to produce the different pilot bunch variants and to facilitate the switching between them. As a proof of principle, the most demanding beam variants have been produced during 2002. The observed stability and reproducibility corresponded to the stringent LHC requirements.

\section{REFERENCES}

[1] M. Benedikt, R. Cappi, C. Carli, G. Cyvoct, M. Giovannozzi, A. Lombardi, D. Manglunki, M. Martini, E. Metral, G. Metral, K. Schindl, M. Vretenar, "Transverse Performance of the Proton Beam Delivered By The PS Complex For The Future LHC”, EPAC 2000, Vienna, June 2000, 1471 (2000).

[2] R. Garoby, S. Hancock, J.-L. Vallet, "Demonstration Of Bunch Triple Splitting In The CERN PS", EPAC 2000, Vienna, June 2000, 304 (2000).

[3] http://psdata.web.cern.ch/psdata/www/icfa9/slides/blas.pdf 